\title{
Das neue NaiS-Anforderungsprofil Steinschlag
}

\author{
Luuk Dorren \\ Frédéric Berger \\ Monika Frehner \\ Markus Huber \\ Kathrin Kühne \\ Roland Métral \\ Arthur Sandri \\ Raphael Schwitter \\ Jean-Jacques Thormann \\ Berchthold Wasser
}

\author{
Hochschule für Agrar-, Forst- und Lebensmittelwissenschaften $(\mathrm{CH})^{*}$ \\ Institut national de recherche en sciences et technologies pour l'environnement et l'agriculture, \\ centre de Grenoble (FR) \\ ETH Zürich, Departement Umweltsystemwissenschaften $(\mathrm{CH})$ \\ Eidgenössische Forschungsanstalt für Wald, Schnee und Landschaft $(\mathrm{CH})$ \\ Hochschule für Agrar-, Forst- und Lebensmittelwissenschaften $(\mathrm{CH})$ \\ Service des forêts et du paysage du canton du Valais, arrondissement du Bas Valais $(\mathrm{CH})$ \\ Bundesamt für Umwelt $(\mathrm{CH})$ \\ Fachstelle für Gebirgswaldpflege $(\mathrm{CH})$ \\ Hochschule für Agrar-, Forst- und Lebensmittelwissenschaften $(\mathrm{CH})$ \\ Natur Dialog $(\mathrm{CH})$
}

\section{Das neue NaiS-Anforderungsprofil Steinschlag}

\begin{abstract}
Neue Erkenntnisse im Bereich Steinschlag und Wald haben dazu geführt, dass das Bundesamt für Umwelt im Jahr 2008 damit begonnen hat, das NaiS-Anforderungsprofil Steinschlag zu überarbeiten. Bislang flossen die Sturzgeschwindigkeit, die Steinmasse sowie die bewaldete Hanglänge im Schutzwald nicht in die Bestimmung des Anforderungsprofils ein. Um alle diese wichtigen Faktoren miteinbeziehen zu können, wurde ein internetbasiertes Formular entwickelt, welches das Anforderungsprofil berechnet. Der vorliegende Artikel gibt einen Überblick über die wichtigsten Prozesse im Steinschlagschutzwald und zeigt auf, auf welchen Grundlagen die Berechnung des neuen Anforderungsprofils basiert. Obwohl nur von Steinschlag gesprochen wird, ist auch bei grossen Blöcken (z.B. $>5 \mathrm{~m}^{3}$ ) eine Waldwirkung gegeben, allerdings wird für eine relevante Energie- und Risikoreduktion ein langes, bewaldetes Transitgebiet mit einer genügenden Anzahl dicker Bäume benötigt. Das neue Anforderungsprofil bietet die Möglichkeit, auch solchen Situationen Rechnung zu tragen.
\end{abstract}

Keywords: protection forest, rockfall, NaiS target profile, silviculture doi: $10.3188 /$ szf.2015.0016

* Länggasse 85, CH-3052 Zollikofen, E-Mail luuk.dorren@bfh.ch

$\mathrm{D}$ er Schutzwald spielt im integralen Risikomanagement von Naturgefahren in der Schweiz eine wichtige Rolle. Er schützt zum Beispiel vor Steinschlag, indem stürzende Steine und Blöcke durch den Aufprall auf die Bäume gebremst oder gar gestoppt werden. Dadurch trägt der Wald an vielen Stellen dazu bei, das durch Steinschlag verursachte Risiko auf ein tragbares Mass zu senken. Dank dem Schutzwald sind technische Massnahmen zur Abdeckung höherer Schutzansprüche oft kostengünstiger (tiefere Installations- oder Unterhaltskosten), und an bestimmten Stellen machen technische Massnahmen überhaupt nur Sinn wegen des zusätzlichen Schutzes durch den Wald (Thali 2006).

Um die Schutzwirkung auf Dauer sicherzustellen, müssen die Wälder in vielen Fällen gepflegt werden. Gründe dafür sind zum Beispiel homogene Bestände mit Verjüngungsmangel, grosse Bestandeslücken, Bestandesüberalterung, Windwurf, Borkenkäfer oder Verbiss durch Huftiere und Weidevieh (Wehrli et al 2007). Grundlage für die Pflege der
Schutzwälder in der Schweiz ist die Vollzugshilfe «Nachhaltigkeit und Erfolgskontrolle im Schutzwald» (NaiS; Frehner et al 2005), in welcher waldbauliche Anforderungsprofile für die verschiedenen Waldstandorte und Naturgefahren beschrieben werden.

Bisher orientierte sich das Anforderungsprofil für Steinschlagschutzwälder lediglich an der Grösse des massgebenden Steins oder Blocks, gegen welchen der Wald Schutz bieten sollte. Es beschrieb einen Waldzustand, der auf wüchsigen Standorten nachhaltig erreicht werden kann und möglichst gut vor Steinschlag schützt. Die Grundlagen fehlten, um ein Anforderungsprofil zu entwickeln, das an die lokale Steinschlaggefährdung angepasst werden konnte. Neue Erkenntnisse zu den Steinschlagprozessen im Wald ermöglichen es nun, den spezifischen Einflussfaktoren besser Rechnung zu tragen. Aus diesem Grund hat das Bundesamt für Umwelt (BAFU) im Jahr 2008 damit begonnen, das NaiS-Anforderungsprofil Steinschlag zu überarbeiten. Dieser Überarbei- 
tungsprozess wurde im Sommer 2014 abgeschlossen, und das neue Anforderungsprofil Steinschlag wurde anschliessend im Internet aufgeschaltet.

Der vorliegende Artikel gibt einen Überblick über die wichtigsten Prozesse im Steinschlagschutzwald und zeigt auf, auf welchen Grundlagen das neue NaiS-Anforderungsprofil basiert.

\section{Kenntnisstand zu den Steinschlag- prozessen im Wald}

Obwohl im Titel des NaiS-Anforderungsprofils nur von Steinschlag gesprochen wird, gilt dieses auch für Blockschlag und sogar Felsstürze. Gemäss Defintion von BRP et al (1997) treten bei Steinschlag Steine mit Durchmessern bis $0.5 \mathrm{~m}$ auf und bei Blockschlag Blöcke mit Durchmessern von $0.5 \mathrm{~m}$ bis zu einem Volumen von $100 \mathrm{~m}^{3}$. Bei grösseren Volumen wird von Felssturz gesprochen. Zur Vereinfachung verwenden wir in diesem Artikel den Begriff Steinschlag für alle relevanten Sturzgefahrenprozesse und den Begriff Stein für alle Sturzkomponenten, unabhängig von ihrer Grösse.

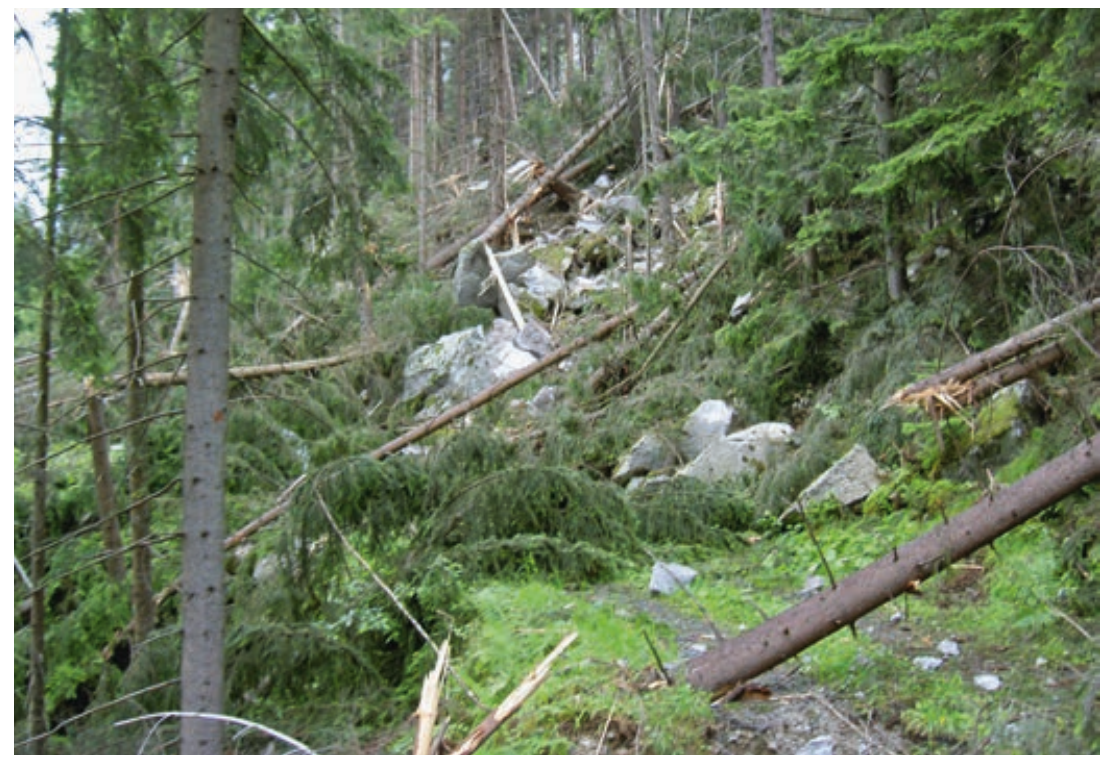

Abb 1 Die Hauptmasse des Sturzmaterials wurde beim Felssturz von 2006 in Gurtnellen im Wilerwald abgelagert. Gemäss Thali (2006) wurden 75\% der 8- m $^{3}$-Steine und 20\% der 20- $m^{3}$-Steine vom Wald zurückgehalten. Die Energiereduktion durch den Wald bei einem 50- $m^{3}-«$ Stein» betrug hingegen lediglich rund $15 \%$.

\begin{tabular}{|c|c|c|c|c|c|c|c|c|}
\hline \multirow[t]{2}{*}{ Distanz vom Startpunkt (m) } & \multicolumn{8}{|c|}{ Anzahl liegende Stämme } \\
\hline & 0 & 1 & 1 & 1 & 1 & 2 & 3 & 4 \\
\hline 40 & & $\bullet$ & & & & ○ & $\bullet$ & - \\
\hline 60 & & & - & & & - & - & - \\
\hline 80 & & & & - & & & - & - \\
\hline 100 & & & & & & & & - \\
\hline Steindurchgänge (\%) & 78 & 15 & 25 & 27 & 29 & 7 & 4 & 4 \\
\hline
\end{tabular}

Tab 1 Wirkung der Anzahl liegender Stämme (Stammdurchmesser $80 \mathrm{~cm}$ ) und von deren Distanz zum Anfang der Sturzbahn (Startpunkt) auf den Anteil der Steine, die gemäss den Modellsimulationen von Berger et al (2014) den Wald durchquerten (Steindurchgänge [\%]). Verwendete Steingrösse: $1 \mathrm{~m}^{3}$.
Auch bei grossen Sturzkomponenten (z.B. $>5 \mathrm{~m}^{3}$ ) ist eine Waldwirkung gegeben (Abbildung 1). Allerdings wird, abhängig von der Höhe der Felswand, für eine relevante Energie- und Risikoreduktion ein langes, bewaldetes Transitgebiet (z.B. >750 m) mit einer genügenden Anzahl grösserer Bäume (z.B. Brusthöhendurchmesser [BHD] $>48 \mathrm{~cm}$ ) benötigt.

Forschung zu Schutzwald und Steinschlag wird seit den 1980er-Jahren betrieben (Couvreur 1982, Jahn 1988, Gsteiger 1993). Sie wurde Ende der 1990erJahre intensiviert, unter anderem im von der Europäischen Union finanzierten Forschungsprojekt ROCKFOR, welches von französischen, österreichischen, schweizerischen und spanischen Forschungsinstituten durchgeführt wurde (Berger et al 2002, Dorren et al 2005, Dorren \& Berger 2005, Perret et al 2004, Brauner et al 2005, Le Hir et al 2006, Stoffel et al 2006, Wehrli et al 2006, Kalberer et al 2007, Lundström et al 2009). Eines der wichtigen Ergebnisse dieses Projekts für das neue NaiS-Anforderungsprofil Steinschlag ist die statistisch relevante Beziehung zwischen der Auslaufdistanz von fallenden Steinen im Wald und der (Bestandes-)Grundfläche, welcher die Steine begegnen (für Details siehe Berger \& Dorren 2007). Diese Beziehung erlaubt es, die Stammzahlverteilung zu berechnen, die benötigt wird, um einen Stein mit einer bestimmten Grösse und einer bestimmten kinetischen Energie innerhalb einer gegebenen Distanz zu stoppen. Weiter haben Steinschlagexperimente im Wald gezeigt, dass das Vorhandensein von Bäumen mit kleinem Durchmesser wichtig ist für eine erhöhte Trefferwahrscheinlichkeit. Dünne Bäume können auch grosse Steine zum Stillstand bringen, sofern diese zuvor auf dicke Bäume gestossen sind (Dorren et al 2005).

Zwischen 2011 und 2014 untersuchten Berger et al (2014) mit Steinschlagexperimenten im Massstab 1:1 und mit Modellsimulationen die Effektivität von liegenden Stämmen und die Auswirkung der Öffnungsgrösse (gemessen von Stammfuss zu Stammfuss) auf die Steinschlaggefährdung unterhalb eines Waldkomplexes. Sie konnten zeigen, dass auch liegende Stämme mit Durchmessern von rund der Grösse des Steindurchmessers die Steinschlaggefährdung signifikant zu reduzieren vermögen (Tabelle 1). Am wirkungsvollsten scheinen dabei Stämme zu sein, die in einem Winkel von ca. 70 schräg zur Falllinie liegen. Wichtig ist, die Stämme möglichst nahe am Entstehungsgebiet zu deponieren, damit sie die Steine bereits während der Beschleunigungsphase bremsen. Um Steine mit liegenden Stämmen zu stoppen, können auch kleinere Verflachungen im Transitgebiet ausgenutzt werden.

\footnotetext{
1 Herunterladbar von www.bafu.admin.ch/naturgefahren > Schutzwald > Nachhaltigkeit im Schutzwald (Projekt NaiS) (1.12.2014)
} 
In Öffnungen von bis 40 m Länge in der Falllinie nimmt die Energie herabstürzender Steine zwar $\mathrm{zu}$, der darunter liegende Waldbestand baut diese aber innerhalb einer Strecke von ungefähr der doppelten Öffnungslänge wieder ab (Berger et al 2014). Sind die Öffnungen grösser, reicht die doppelte Öffnungsgrösse nicht mehr aus, um die Energiezunahme wieder abzubauen. Weil die Energiezunahme mit der Geländeneigung steigt, ist der Effekt bei steileren Hängen $\left(>35^{\circ}\right)$ grösser als bei flacheren $\left(<30^{\circ}\right)$. Weiter zeigten Berger et al (2014), dass es wichtig ist, Öffnungen direkt unterhalb des Entstehungsgebiets so klein wie möglich zu halten.

Zusammenfassend kann der Steinschlagprozess im Wald vereinfacht als eine Bilanz zwischen energieerhöhenden und energiemindernden Faktoren betrachtet werden. Die energieerhöhenden Faktoren sind die Sturzgeschwindigkeit (bestimmt durch die initiale Fallhöhe des Steins sowie die Steilheit des Geländes) und die Steinmasse (bestimmt durch die Form, die Steingrösse und die Gesteinsdichte). Gemindert wird die Sturzenergie durch die Gesamtmasse an Holz, welche einem Stein auf seiner Sturzbahn begegnet, und die Energieaufnahmekapazität der Bäume (bestimmt durch die Baumart; Dorren \& Berger 2005). Die Gesamtmasse an Holz, welche einem Stein auf seiner Sturzbahn begegnet, ist abhängig von der bewaldeten Hanglänge sowie der Stammzahl und der Durchmesserverteilung im Bestand. Sie kann durch die Grundfläche beschrieben werden.

\section{Bedürfnis zur Verbesserung des alten Anforderungsprofils}

Bislang wurden die Sturzgeschwindigkeit und die Steinmasse (Gefahrenpotenzial) sowie die bewaldete Hanglänge und die Baumart (Schutzpotenzial) im NaiS-Anforderungsprofil für die Bestimmung der notwendigen Stammzahlen nicht berücksichtigt. Gerade aber die bewaldete Hanglänge beeinflusst die räumliche Ordnung und die Art der Eingriffe und damit den waldbaulichen Handlungsspielraum massgeblich (Zürcher et al 2010).

Daneben wurde das bisherige Nais-Anforderungsprofil in Bezug auf die vorgegebene maximale Öffnungsgrösse in der Falllinie oft als zu restriktiv

\begin{tabular}{|l|l|c|}
\hline Waldtyp & Verwendete Kriterien & $\begin{array}{c}\mathbf{G}_{\max } \\
\left(\mathrm{m}^{2} / \mathbf{h a}\right)\end{array}$ \\
\hline Buchenmischwald & $\begin{array}{l}\text { Buchenanteil }<80 \% \text { und } \\
\text { Nadelbaumanteil <30\% }\end{array}$ & 40 \\
\hline Buchenwald & $\begin{array}{l}\text { Buchenanteil }>80 \% \text { und } \\
\text { Nadelbaumanteil }<30 \%\end{array}$ \\
\hline Übrige Wälder & Nadelbaumanteil $>30 \%$ & 45 \\
\hline
\end{tabular}

Tab 2 Maximale Grundflächen $\left(G_{\max }\right)$ für drei verschiedene Waldtypen. Das Internettool rechnet diese nachhaltig möglichen Grundflächen in Abhängigkeit von den tatsächlich vorhandenen Baumarten. empfunden (von Büren 2011), weil sich das Schaffen guter Verjüngungsverhältnisse unter Einhaltung des Anforderungsprofils als schwierig oder gar unmöglich erwies (Aeberli 2012). Es wurde denn auch häufig diskutiert, ob sich mit zusätzlichen Massnahmen (z.B. hohe Stöcke oder liegende Stämme) eine grössere Öffnung in der Falllinie ermöglichen liesse.

\section{Das neue Anforderungsprofil und seine Grundlagen}

Das neue NaiS-Anforderungsprofil betrachtet nicht nur die massgebende Steingrösse, sondern auch die Sturzenergie der Steine sowie die bewaldete Hanglänge. Mithilfe eines Internettools (siehe nächstes Kapitel) kann ein jeweils an die lokalen Gegebenheiten angepasstes Minimal- und Idealprofil berechnet werden. Basis des Tools ist die Methode Rockfor. net von Berger \& Dorren (2007), welche abgeändert und verbessert wurde, um den Anforderungen der Praxis gerecht zu werden. Das Internettool gibt die Stammzahlverteilung für das Minimal- und das Idealprofil aus und schätzt die Schutzwirkung des jeweiligen Waldes gegen Steinschlag ab. Die grundlegenden Überlegungen zur Berechnung der Profile werden nachstehend skizziert.

\section{Grundfläche und Stammzahlverteilung gemäss Minimal- und Idealprofil}

Die Berechnung der Stammzahlverteilung für das Minimal- und Idealprofil basiert auf der Ermittlung derjenigen Grundfläche, die benötigt wird, um die Sturzenergie des definierten Steines innerhalb der definierten bewaldeten Hanglänge zu vernichten. In Ergänzung zur ursprünglichen Herleitung in Rockfor.net (Berger \& Dorren 2007) werden heute folgende Faktoren miteinbezogen:

- Die theoretische Grundfläche $\left(G_{\text {theo }}\right)$ ist diejenige Grundfläche, die einem Stein auf seiner Sturzbahn begegnen muss, um die Energie mit einer minimalen Anzahl Aufpralle vernichten zu können (alle 43 m ein wirksamer Aufprall; Standardwert in Rockfor.net).

- G $G_{\text {theo }}$ wird für das Minimalprofil verwendet, für das Idealprofil wird ein Sicherheitszuschlag von $5 \mathrm{~m}^{2}$ Grundfläche zugegeben.

- Weil $\mathrm{G}_{\text {theo }}$ die im Wald nachhaltig mögliche Grundfläche weit übertreffen kann, wurden, unter anderem auf Basis von Daten des Landesforstinventars (Messmer 2014), realistische Maximalwerte $\left(G_{\max }\right)$ als Obergrenze definiert. Diese sind im Tool abhängig von den vorhandenen Baumarten (Tabelle 2). Für das Idealprofil gilt $\mathrm{G}_{\max }$ als Obergrenze, für das Minimalprofil werden von diesen Werten $5 \mathrm{~m}^{2}$ abgezogen.

- Als untere Begrenzung des Minimalprofils wurde eine Grundfläche von $20 \mathrm{~m}^{2}$ festgelegt. Sie ist 


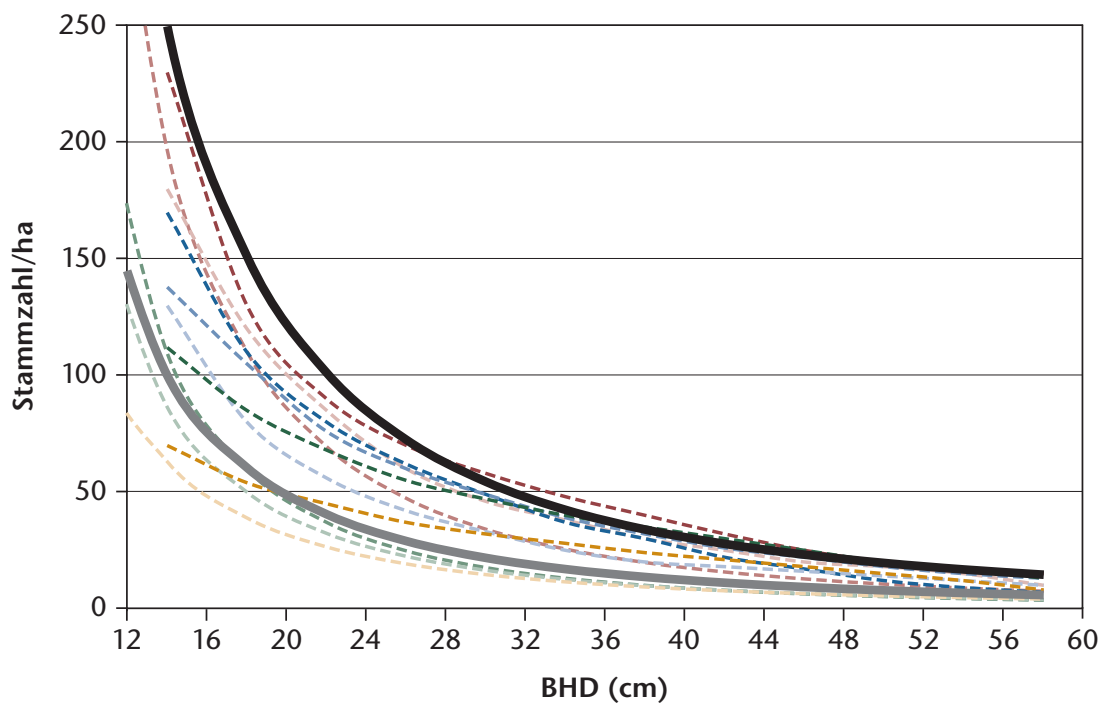

Basiskurve mit $\mathrm{G}=50 \mathrm{~m}^{2} / \mathrm{ha}$

Basiskurve mit $\mathrm{G}=20 \mathrm{~m}^{2} / \mathrm{ha}$

- - - LFI-Messmer (2014), Buchenw. B_gut $(n=143)$ / Tannen-Fichtenw. B_gut $(n=165)$

- - - Burkhalter (2013), Untermontan $(\mathrm{N}=3$ )

- = LFI-Messmer (2014), Buchenmischwald B_gering $(\mathrm{n}=45)$

- - - LFI-Messmer (2014), Tannen-Buchenwald B_mäss $(n=167)$

- - - LFI-Messmer (2014), Tannen-Fichtenwald $B$ mäss $(n=323)$

LFI-Messmer (2014), Fichtendom. Hochm. B_mäss $(\mathrm{n}=117)$

Abb 2 Stammzahlverteilung der Basiskurven mit Grundfläche (G) $20 \mathrm{~m}^{2}$ und $50 \mathrm{~m}^{2}$ sowie Trendlinien mit Daten real beobachteter Wälder (Burkhalter 2013, Messmer 2014, Schütz 1997, Zingg 2012). B_ ist ein Mass für die Bonität (sehr gut, gut, mässig, gering), N gibt die Anzahl analysierter Bestände wieder und n die Anzahl verwendeter LFI-Probeflächen.

damit deutlich tiefer als im bisherigen Minimalprofil, das eine minimale Grundfläche von 30 bis $40 \mathrm{~m}^{2}$ voraussetzte. Die vom BAFU zur Weiterentwicklung von NaiS eingesetzte Arbeitsgruppe liess sich bei dieser Änderung von der Überlegung leiten, dass in gewissen Fällen ein Wald mit $20 \mathrm{~m}^{2}$ Grundfläche ausreichend Schutz zu bieten vermag, dass aber in stufigen Beständen diese Grundfläche nur einer Stammzahl von etwa 200 Bäumen pro Hektare mit einem BHD von mehr als $12 \mathrm{~cm}$ entspricht.

- Bei der Berechnung der aufzunehmenden Sturzenergien durch die Bäume berücksichtigt das Tool die vorhandene Baumartenzusammensetzung, mit einer Ausnahme. Um zu verhindern, dass für Steine mit kleiner Sturzenergie im Laubwald viel tiefere Grundflächen als in Nadelwäldern berechnet werden, wird auf diese Differenzierung verzichtet, wenn die Sturzenergie des Steines kleiner ist als die maximale Energie, die von einer Fichte der gleichen Grundfläche aufgenommen werden kann. In diesem Fall wird nur mit der Energieaufnahmekapazität einer Fichte, der Baumart mit der geringsten Energieaufnahmekapazität im Tool, gerechnet.

Nach Berechnung der Grundflächen von Idealund Minimalprofil werden daraus die Stammzahlverteilungen, die sogenannten Basiskurven, ausgegeben. Dafür werden die Grundflächen gleichmässig über alle BHD-Klassen verteilt - d.h., jede BHD-Klasse hat einen gleichen Anteil an der Grundfläche. Dieses Vorgehen wurde gewählt, um die Stammzahlverteilung stufiger Bestände nachzubilden. Der Vergleich mit realen stufigen Beständen (Schütz 1997, Zingg 2012, Burkhalter 2013, Messmer 2014) zeigt, dass die Basiskurven sich denn auch im Bereich der Stammzahlverteilungen realistischer Bestände bewegen.

Da grössere Steine am effizientesten gestoppt werden können, wenn auch dicke Bäume vorhanden sind, und bei kleineren Steinen eine hohe Stammzahl günstig ist, wurden für verschiedene Steinvolumen unterschiedliche maximale BHD-Klassen festgelegt. Wenn das Volumen des massgebenden Steines grösser als $0.2 \mathrm{~m}^{3}$ ist, werden die Klassen 10 $(8-12 \mathrm{~cm})$ bis $58(56-60 \mathrm{~cm})$ verwendet. Bei Volumen unter $0.05 \mathrm{~m}^{3}$ ist die maximale BHD-Klasse gleich $38(36-40 \mathrm{~cm})$, bei Steinen mit Volumen zwischen 0.05 und $0.2 \mathrm{~m}^{3}$ ist es Klasse $46(44-48 \mathrm{~cm})$.

\section{Schutzwirkung des Waldes}

Die Schutzwirkung des Waldes ist ein Mass für den Anteil der vom Wald gestoppten Steine. Es entspricht dem Verhältnis zwischen der effektiven Grundfläche des betrachteten Waldkomplexes und der erforderlichen Grundfläche $G_{\text {theo }}$. Mit dem Verhältnis zwischen vorhandener und benötigter Stammzahl $\left(\mathrm{N}_{\mathrm{ist}} / \mathrm{N}_{\text {soll }}\right)$ wird dann die berechnete Schutzwirkung korrigiert. So wird vermieden, dass für Wälder mit genügender Grundfläche, aber ungenügender Stammzahl eine gute Schutzwirkung ausgegeben wird. Weil für das im Tool verwendete Modell vereinfachende Annahmen getroffen werden mussten (z.B. stark vereinfachte Topografie, Vernachlässigung der Oberflächenbeschaffenheit, keine Berücksichtigung von Steinschlagschneisen), wird die Schutzwirkung nur in den folgenden Klassen ausgegeben: 0-25\%, 25-50\%, 50-75\% (sofern $\mathrm{N}_{\text {ist }} / \mathrm{N}_{\text {soll }}$ $>0.5$ ), $75-95 \%$ (sofern $\mathrm{N}_{\mathrm{ist}} / \mathrm{N}_{\text {soll }}>0.6$ ) und $95-99 \%$ (sofern $\mathrm{N}_{\mathrm{ist}} / \mathrm{N}_{\text {soll }}>0.8$ ). Eine hundertprozentige Wirkung wird vom Tool nicht ausgegeben, weil ein hundertprozentiger Schutz von keiner Schutzmassnahme gewährleistet werden kann.

Wichtig ist, dass die vom Tool ermittelte Schutzwirkung immer nur im Zusammenhang mit der notwendigen Risikoreduktion beim Schadenpotenzial beurteilt wird. Es kann auch eine geringe Schutzwirkung des Waldes ausreichen, um das Risiko auf ein tragbares Mass zu senken. So kann entlang von Strassen eine Schutzwirkung von beispielsweise $25 \%$ reichen, um ein individuelles Todesfallrisiko von weniger als $10^{-5} \mathrm{zu}$ erhalten. Eine isolierte Betrachtung einer errechneten tiefen Schutzwirkung ist daher kaum sinnvoll, vielmehr sind in solchen Fällen detaillierte Risikoanalysen nötig. Bei einer berechneten hohen Schutzwirkung kann davon ausgegangen werden, dass der betrachtete Waldkomplex guten Schutz gegen das analysierte Steinschlagereig- 


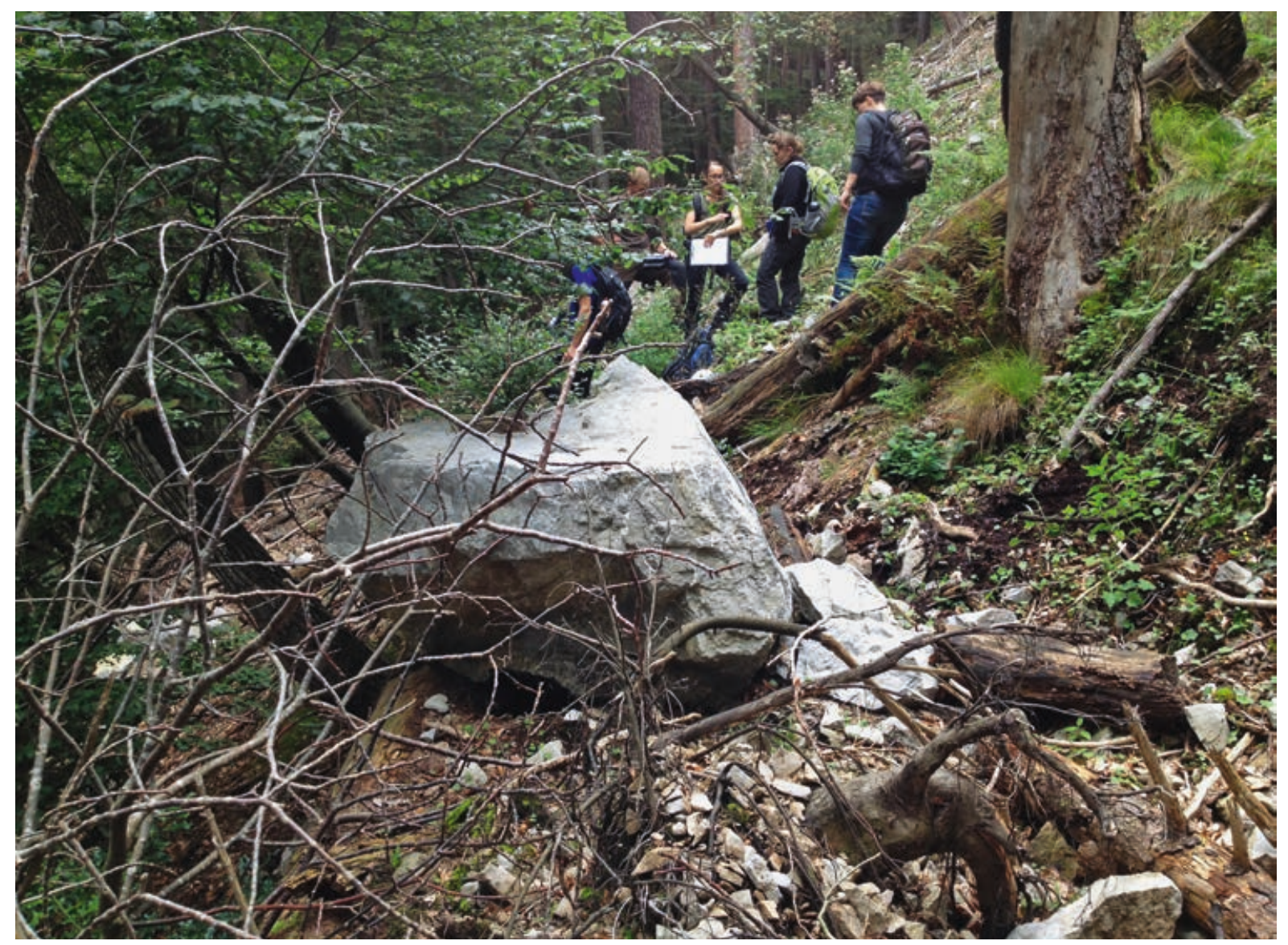

Abb 3 Dieser $1 \mathrm{~m}^{3}$ grosse Stein wurde von einem alten liegenden Stamm im Salzacherwald (Merligen BE) gestoppt. Die übrigen Splitter (mehrere bis zu $0.5 \mathrm{~m}^{3}$ ) wurden alle von stehenden Bäumen aufgehalten.

nis zu leisten vermag. Eine gute Datengrundlage ist die Voraussetzung für gute Ergebnisse bei der Berechnung der Schutzwirkung.

\section{Grössere Öffnungen und zusätzliche Massnahmen}

Auf Basis der Ergebnisse von Berger et al (2014) wurden für das Anforderungsprofil folgenden Kriterien für Öffnungen festgelegt:

- maximaler Stammabstand in der Falllinie $<40 \mathrm{~m}$

- bei Öffnungen grösser als $20 \mathrm{~m}$ in der Falllinie: hohe Stöcke (ca. $1.30 \mathrm{~m}$ ) sowie alle zehn Meter mindestens zwei liegende Stämme (Abbildung 3) mit einem Durchmesser von mindestens dem Steindurchmesser und schräg zur Falllinie (am wirkungsvollsten mit einem Winkel von ca. $70^{\circ}$ ) platziert.

Hierzu ist zu erwähnen, dass Öffnungen grösser als $20 \mathrm{~m}$ nur geschlagen werden sollten, wenn sie für die Verjüngung zwingend erforderlich sind. Liegende Stämme haben nur während einer beschränkten Zeit (max. ca. 10 Jahre bei Buchen und max. ca. 25 Jahre bei Fichten) eine gute Wirkung. Das liegende Holz muss wie alle anderen Schutzbauten periodisch kontrolliert und unterhalten werden. Gefährliche Steine müssen sicher deponiert oder allenfalls kontrolliert gesprengt werden.

\section{Das Internettool ...}

Zur Ermittlung des entsprechenden Anforderungsprofils wurde ein internetbasiertes $\mathrm{Tool}^{2}$ entwickelt.

Es präsentiert die benötigte Stammzahlverteilung auf zwei unterschiedliche Arten (Abbildung 4). Einerseits grafisch, wobei der obere Rand der roten Balken jeweils dem Idealprofil, der untere Rand dem Minimalprofil entspricht. Andererseits werden die für die BHD-Klassen 8-12 cm, 12-24 cm, 24-36 cm und $\geq 36 \mathrm{~cm}$ benötigten Stammzahlen aufgelistet. Die Angaben zur BHD-Klasse $8-12 \mathrm{~cm}$ wurden ergänzt aufgrund ihrer Relevanz im Steinschlagschutzwald.

Damit ein Wald bei einer Länge des Transitgebiets von weniger als $75 \mathrm{~m}$ Steine überhaupt wirksam stoppen kann, ist eine hohe Stammzahl für eine hohe Trefferwahrscheinlichkeit notwendig. Bei niedriger Stammzahl auf so kurzer Distanz ist die Chance gross, dass der Stein ohne Baumtreffer durchgeht. Niederwälder weisen in der Regel eine hohe Stammzahl auf (Jancke et al 2009). Daher wird im Internettool ein Niederwaldprofil vorgeschlagen, wenn die bewaldete Hanglänge kürzer als $75 \mathrm{~m}$ ist und nur Laubbaumarten vorkommen.

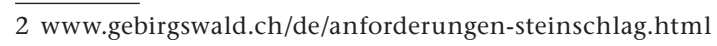
$(1.12 .2014)$ 


\section{Minimal- und Idealprofil für die Schutzwirkung gegen Steinschlag:}

Stammzahlen für das NaiS-Formular 2:

Benötigte Stammzahl mit BHD 8-12 cm: 390 (minimal) bis 490 (ideal) St./ha Benötigte Stammzahl mit BHD 12-24 cm: 400 (minimal) bis 500 (ideal) St./ha Benötigte Stammzahl mit BHD 24-36 cm: 140 (minimal) bis 170 (ideal) St./ha Benötigte Stammzahl mit BHD .36 cm: 110 (minimal) bis 140 (ideal) St./ha

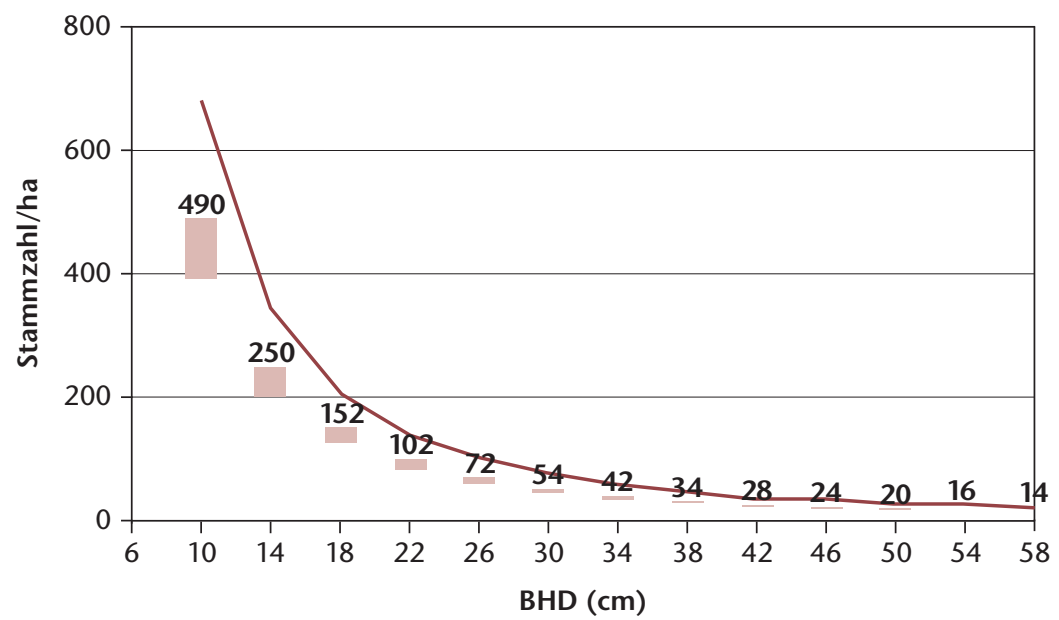

Nachhaltiges Profil — Profil der maximalen Energievernichtung

- Nachhaltige Grundfläche (ab $8 \mathrm{~cm} \mathrm{BHD):} 45 \mathrm{~m}^{2} / \mathrm{ha}$ (Minimalprofil) bis $50 \mathrm{~m}^{2} /$ ha (Idealprofil; $\mathrm{G}_{\max }$ )

- Grundfläche (ab $8 \mathrm{~cm}$ BHD) für eine maximale Energievernichtung (damit möglichst alle Steine gestoppt werden; $\mathrm{G}_{\text {theo }}$ ): $68 \mathrm{~m}^{2} / \mathrm{ha}$

\section{Aktuelle Schutzwirkung des Waldes: $50-75 \%$}

Abb 4 Ergebnisdarstellung im Internettool. Die rote Linie zeigt ausgehend von der theoretisch erforderlichen Grundfläche $\left(G_{\text {theo }}\right)$ die Stammzahlverteilung für die maximale Energievernichtung. Diese ist im analysierten Schutzwald nicht auf Dauer erreichbar. Die rosa Balken zeigen das realistische, nachhaltige Profil, welches anhand der maximalen, nachhaltigen Grundfläche $\left(G_{\max }\right)$ ermittelt wurde.

\section{... und seine Anwendung}

Zur Anwendung des Internettools sind Angaben zum Stein, zum Hang sowie zu den Baumarten notwendig (Tabelle 3). Die Angaben zu den Stammzahlen beziehungsweise zur mittleren Stammzahl und zur Grundfläche dienen nur dazu, die gegenwärtige Schutzwirkung eines Waldkomplexes zu beurteilen. Diese Beurteilung gilt für die ganze bewaldete Hanglänge. Das bedeutet, dass die Eingabedaten die ganze bewaldete Fläche zwischen Entstehungsgebiet und Schadenpotenzial repräsentieren müssen. Die Anwendung des neuen Profils erfordert somit eine vertiefte Analyse des gesamten Schutzwaldkomplexes. Die Angaben zum Minimal- und Idealprofil sind ebenfalls für den ganzen Komplex und nicht für einzelne Bestände gültig. Die heutigen Bestände können jedoch mit diesen Angaben verglichen und es kann der Handlungsbedarf pro Hangabschnitt oder Bestand festgelegt werden. So können mittels Bewirtschaftung über längere Zeit die erforderlichen Eigenschaften für einen gesamten Waldkomplex erreicht beziehungsweise erhalten werden.

Für die Bestimmung des Anforderungsprofils eines Waldkomplexes sollten die dem NaiS-Standort entsprechenden Baumartenanteile eingegeben werden, für die Bestimmung der heutigen Schutzwirkung hingegen die real beobachteten Baumartenanteile. Falls ein ganzer Hang ähnlich bestockt ist, können Bestandeskennzahlen zum Beispiel aus Stichprobeninventuren direkt übernommen werden. Stehen entlang der potenziellen Sturzbahn aber
Eingangsgrösse

\section{Beschreibung des Steines}

\section{Steingrössen (in $\mathrm{m}$ )}

Gesteinsdichte (in $\mathrm{kg} / \mathrm{m}^{3}$ )

Form des Steines

\section{Beschreibung des Hanges}

Mittlere Hangneigung (in ${ }^{\circ}$ )

Höhe der Felswand (in m)

Bewaldete Hanglänge (in m)

Hanglänge unbewaldet (in m)

\section{Beschreibung des Waldes}

Baumartenanteile (in \%)*

\section{Zusätzlich (für Berechnung der Schutzwirkung)}

Stammzahl mit BHD 8-12 cm (St./ha)

Stammzahl mit BHD 12-24 cm (St./ha)

Stammzahl mit BHD 24-36 cm (St./ha)

Stammzahl mit BHD $\geq 36 \mathrm{~cm}$ (St./ha)

oder

mittlere Stammzahl (St./ha) und Grundfläche (in $\mathrm{m}^{2} / \mathrm{ha}$ )
Bemerkung

Höhe, Breite und Tiefe des massgebenden Steins

Beispielwerte werden im Internettool vorgeschlagen

Rechteckig, Ellipsoid, Kugel oder Scheibe

Horizontal gemessen

Zwischen Felswandfuss und Obergrenze des bewaldeten Transitgebiets; horizontal gemessen.

Fichte, Tanne, Buche, übrige Laubbäume, übrige Nadelbäume.

Der Prozentsatz entspricht dem Anteil der Stämme.

Diese Angaben werden nur für die Berechnung der Schutzwirkung des heutigen Waldes benötigt

Tab 3 Die benötigten Eingangsgrössen zur Berechnung des Anforderungsprofils im Internettool. * Für die Bestimmung des Anforderungsprofils sind die dem NaiS-Standort entsprechenden Baumartenanteile einzugeben, für die Bestimmung der heutigen Schutzwirkung die aktuellen Baumartenanteile. 
sehr unterschiedliche Bestände (z.B. Stangen- und starke Baumhölzer), müssen die Kennzahlen pro Bestand erhoben und entsprechend ihrer Strecke an der Sturzbahn gewichtet werden. Bei dieser Mittelwertbildung müssen auch Lücken berücksichtigt werden. Dabei sind Jungwaldflächen mit mittlerem BHD $<8 \mathrm{~cm}$ wie Lücken zu behandeln.

Die korrekte Bestimmung der Hangneigung im Transitgebiet ist manchmal schwierig, weil der Übergang vom Entstehungs- zum Transitgebiet fliessend sein kann. In diesem Fall wird die Hangneigung am besten mithilfe der topografischen Karte bestimmt, und zwar mit dem Verhältnis zwischen der horizontalen und vertikalen Distanz von der Oberkante bis zur Unterkante des Transitgebiets.

Eine Frage, die bei der Anwendung des NaisAnforderungsprofils Steinschlag immer wieder gestellt wird, ist, wie sich die Grösse des massgebenden Steins bestimmen lasse. Die weitaus wichtigere Frage wäre: Wovor will man sich schützen? Der Ereigniskataster, sofern vorhanden, ist die wichtigste Grundlage zu ihrer Beantwortung. Normalerweise gibt er nicht nur Auskunft über die Steingrössen, sondern auch über die Häufigkeit von Sturzprozessen. Über Informationen dazu verfügen oft auch Strassenämter (Tiefbauämter) sowie Bahngesellschaften, weil Sturzereignisse den Betrieb der Infrastruktur direkt beeinträchtigen. Falls keine Informationen vorliegen, muss man sich auf stumme Zeugen (abgelagerte Steine, vor allem frischere Steine, die nicht vollständig mit Moos überwachsen oder völlig im Hang eingebettet sind, oder auf Kluftkörper im Fels im Entstehungsgebiet) verlassen.

Nicht überall, wo Gefahrenhinweiskarten (z.B. SilvaProtect) Steinschlag ausweisen, tritt solcher auch tatsächlich auf. In Wäldern ohne frische oder zugewachsene Schlagspuren an den Bäumen oder ohne abgelagerte Steine nahe am Entstehungsgebiet soll deshalb die Steinschlagaktivität hinterfragt werden. In solchen Fällen könnte es sein, dass Steinschlag nicht der primäre Naturgefahrenprozess im Waldkomplex ist. Allenfalls müssten dort andere NaiS-Anforderungsprofile (z.B. Rutschungen oder Lawine) angewendet werden. Eventuell kommt man auch zum Schluss, dass der Wald wegen des fehlenden Gefahrenpotenzials gar kein Schutzwald ist.

Es gibt Fälle, in denen das neue Anforderungsprofil höhere Stammzahlen fordert als das alte Profil. Wenn aber eine waldbauliche Flexibilität gegeben ist aufgrund niedriger Energien oder langer bewaldeter Hänge, wird das Tool diese in niedrigeren Stammzahlen widerspiegeln.

\section{Ausblick}

Das neue Nais-Anforderungsprofil Steinschlag zielt auf stufige Strukturen im Steinschlagschutz- wald ab. Wie bisher ist das Idealprofil als Zukunftsziel zu sehen; die Massnahmen werden auf dieses Ziel hin ausgerichtet. Abhängig vom Ausgangsbestand kann dieses Ziel kurz-, mittel-, aber oft auch erst langfristig erreicht werden. Die Begründung für die definierten Anforderungen liegt in der nachhaltigen Struktur, der genügenden Grundfläche und der damit dauernd optimalen Schutzwirkung von solchen Wäldern.

Bäume mit kleinen Durchmessern sind enorm wichtig für eine erhöhte Trefferwahrscheinlichkeit. Sie sind oft der Grund dafür, dass auch grosse Steine zum Stillstand kommen, nachdem sie auf dicke Bäume gestossen sind. Steinschlagexperimente im Wald (Berger \& Dorren 2007) haben dies klar gezeigt. Dies ist ein weiteres Argument für den stufigen Aufbau von Steinschlagschutzwäldern. Auch wenn sie heute (noch) nicht genau der klassischen Plenterwaldstruktur entsprechen, entfalten sie trotzdem kurz- und langfristig die bestmögliche Schutzwirkung.

Das neue Nais-Anforderungsprofil Steinschlag kann seit August 2014 angewendet werden. Die Erfahrungen beim Einsatz in der Praxis werden zeigen, ob sich das entwickelte Internettool als Lösung zur Umsetzung des neuesten Kenntnisstands im Steinschlagschutzwald bewähren kann.

Eingereicht: 7. Oktober 2014, akzeptiert (mit Review): 29. November 2014

\section{Literatur}

AEBERLI C (2012) Eignung neuer Kriterien zur Beurteilung der Schutzwirkung von Bergwäldern vor Steinschlag (NaiS-Anforderungsprofil). Eine Simulations- und Feld-Praktikabilitätsstudie in der Zentralschweiz. Zollikofen: Hochschule Agrar- ForstLebenswissenschaften, Bachelorarbeit. $81 \mathrm{p}$.

BERGER F, QUETEL C, DORREN LKA (2002) Forest: A natural protection means against rockfalls, but with which efficiency? The objectives and methodology of the rockfor project. In: Proc Interpraevent, Pacific Rim, Matsumoto (Japan). pp. 815826.

BERGER F, DORREN LKA (2007) Principles of the tool Rockfor.net for quantifying the rockfall hazard below a protection forest. Schweiz Z Forstwes 158: 157-165. doi: 10.3188/szf.2007.0157

BERGER F, BOURRIER F, OLMEDO-MANICH I, BIGOT C (2014) Convention OFEV-Irstea 2011-2014, Rapport final. Grenoble: Centre régional Irstea. $90 \mathrm{p}$.

BRAUNER M, WEINMEISTER W, AGNER P, VOSPERNIK S, HOESLE B (2005) Forest management decision support for evaluating forest protection effects against rockfall. For Ecol Manage 207: 75-85.

BRP, BWW, BUWAL (1997) Berücksichtigung der Massenbewegungsgefahren bei raumwirksamen Tätigkeiten. Empfehlungen 1997. Bern: Bundesamt Raumplanung. 42 p.

BURKHALTER S (2013) Optimale Steinschlagschutzwälder gemäss NaiS-Anforderungen - Charakterisierung und Analyse. Zollikofen: Hochschule Agrar- Forst- Lebenswissenschaften, Bachelorarbeit. $85 \mathrm{p}$.

COUVREUR S (1982) Les forêts de protection contre les risques naturels. Nancy: Ecole nationale du génie rural, des eaux et forêts. 89 p. 
DORREN LKA, BERGER F (2005) Stem breakage of trees and energy dissipation during rockfall impacts. Tree Physiol 26: 63-71.

DORREN LKA, BERGER F, LE HIR C, MERMIN E, TARDIF P (2005) Mechanisms, effects and management implications of rockfall in forests. For Ecol Manage 215: 183-195.

FREHNER M, WASSER B, SCHWITTER R (2005) Nachhaltigkeit und Erfolgskontrolle im Schutzwald. Wegleitung für Pflegemassnahmen in Wäldern mit Schutzfunktion. Bern: Bundesamt Umwelt Wald Landschaft. 564 p.

GSTEIGER P (1993) Steinschlagschutzwald. Ein Beitrag zur Abgrenzung, Beurteilung und Bewirtschaftung. Schweiz Z Forstwes 144: 115-132.

JAHN J (1988) Entwaldung und Steinschlag. In: Proc Interpraevent, Graz, vol 1. pp. 185-198.

JANCKE O, DORREN LKA, BERGER F, FUHR M, KÖHL M (2009) Implications of coppice stand characteristics on the rockfall protection function. For Ecol Manage 259: 124-131.

KALBERER M, AMMANN M, JONSSON M (2007) Mechanische Eigenschaften der Fichte: Experimente zur Analyse von Naturgefahren. Schweiz Z Forstwes 158: 166-175. doi: 10.3188/ szf.2007.166

LE HIR C, DIMNET E, BERGER F (2006) Etude de la trajectographie des chutes de blocs en forêts de montagne. Bull Lab Ponts Chaussées 263/264: 85-101.

LUNDSTRÖM T, JONSSON MJ, VOLKWEIN A, STOFFEL M (2009) Reactions and energy absorption of trees subject to rockfall: a detailed assessment using a new experimental method. Tree Physiol 29: 345-359.

MESSMER C (2014) Grundflächen im Steinschlagschutzwald. Ein Vergleich mit LFI3-Daten. Zürich: ETH Zürich, Departement Umweltsystemwissenschaften, Masterarbeit. 129 p.
PERRET S, DOLF F, KIENHOLZ H (2004) Rockfalls into forests: Analysis and simulation of rockfall trajectories - considerations with respect to mountainous forests in Switzerland. Landslides 1: 123-130.

SCHÜTZ JP (1997) Sylviculture 2: la gestion des forêts irrégulières et mélangées. Lausanne: Presses polytechniques et universitaires romandes. $178 \mathrm{p}$.

STOFFEL M, WEHRLI A, KÜHNE R, DORREN LKA, PERRET S ET AL (2006) Assessing the protective effect of mountain forests against rockfall using a 3D simulation model. For Ecol Manage 225: 113-222.

THALI U (2006) Schutzwirkung des Waldes anhand des FelssturzEreignisses «Wilerwald», Gurtnellen, vom 31. Mai 2006. Altdorf: Kanton Uri, Bericht Schweiz Nationalstrassen N2. 21 p.

VON BÜREN M (2011) Pflege von Steinschlagschutzwäldern. Auswirkungen des Steinschlag-Anforderungsprofils auf die Holzernteeingriffe im Steinschlagschutzwald. Zollikofen: Schweizerische Hochschule Landwirtschaft, Bachelorarbeit. 52 p.

WEHRLI A, DORREN LKA, BERGER F, ZINGG A, SCHÖNENBERGER W ET AL (2006) Modelling the long-term effects of forest dynamics on the protective effect against rockfall. For Snow Landscape Res 80: 57-76.

WEHRLI A, BRANG P, MAIER B, DUC P, BINDER F ET AL (2007) Schutzwaldmanagement in den Alpen - eine Übersicht. Schweiz Z Forstwes 158: 142-156. doi: 10.3188/szf.2007.0142 ZINGG A (2012) Überall plentern? Wald Holz 93 (9): 24-28.

ZÜRCHER K, WIEDMER Y, THORMANN JJ (2010) Weiterentwicklung NaiS-Anforderungsprofil Steinschlag: minimale bewaldete Hang- und kritische Lückenlänge. Schlussbericht. Zollikofen: Schweizerische Hochschule Landwirtschaft. 36 p.

\section{Le nouveau profil d'exigences pour chutes de pierres}

Pour donner suite à de nouvelles connaissances et expérimentations au niveau des chutes de pierres en forêt, l'Office fédéral de l'environnement a commencé en 2008 à adapter le profil d'exigences pour les chutes de pierres du classeur Gestion durable des forêts de protection (NaiS). L'ancien profil n'a pas pris en compte la vitesse de propagation d'un bloc, sa masse ainsi que la longueur boisée du versant. Afin d'intégrer tous ces facteurs importants, un utilitaire internet a été élaboré. Il permet de calculer le profil d'exigences. Le présent article mentionne les processus principaux qui se déroulent en forêt de protection et précise les bases de calcul utilisées pour établir le profil d'exigences. Même si l'on parle uniquement de chutes de pierres, la forêt peut avoir aussi un effet positif envers de gros blocs, dépassant par exemple les $5 \mathrm{~m}^{3}$. L'efficacité d'un peuplement pour réduire d'une manière significative l'énergie d'un tel bloc, et par là même le risque, dépend de la zone de transit, en particulier de sa longueur et de la présence d'arbres de gros diamètre. Le nouveau profil d'exigences permet aussi de prendre en compte de telles situations.

\section{The new NaiS target profile for rockfall}

Due to new findings on the protective function of forests against rockfall, the Federal Office for the Environment started in 2008 with a revision of the Swiss target profiles for rockfall protection forests. Until now, fall velocity of rocks, rock mass, as well as the forested slope length, were not considered for the determination of the target profile. In order to incorporate all of these important factors, an Internet-based form has been developed which calculates the target profile. This article firstly gives a short overview of the most important aspects of rockfall in protection forests and secondly presents the approach used for the calculation of the new target profile. Although the German word "Steinschlag" only refers to small rock particles, forests also have a protective effect against large blocks (e.g. $>5 \mathrm{~m}^{3}$ ), however, a long forested transit zone with a sufficient number of trees with larger stem diameters is required for a relevant energy and risk reduction. The new target profile offers possibilities to account for such situations as well. 